\title{
Agôn
}

Revue des arts de la scène

Critiques | Saison 2016-2017

\section{Avignon, 6 \& 7 juillet : Antigone, de Sophocle, mise en scène Satoshi Miyagi}

Antigone, tragédie vs cérémonie

\section{Caroline Châtelet}

\section{OpenEdition}

Journals

Édition électronique

URL : http://journals.openedition.org/agon/4036

DOI : 10.4000 /agon.4036

ISSN : 1961-8581

Éditeur

Association Agôn

Référence électronique

Caroline Châtelet, «Avignon, 6 \& 7 juillet : Antigone, de Sophocle, mise en scène Satoshi Miyagi », Agôn [En ligne], Critiques, mis en ligne le 12 juillet 2017, consulté le 23 septembre 2020. URL : http://

journals.openedition.org/agon/4036; DOI : https://doi.org/10.4000/agon.4036

Ce document a été généré automatiquement le 23 septembre 2020.

Association Agôn et les auteurs des articles 


\section{Avignon, 6 \& 7 juillet : Antigone, de Sophocle, mise en scène Satoshi Miyagi}

Antigone, tragédie vs cérémonie

\section{Caroline Châtelet}

\section{RÉFÉRENCE}

Antigone de Sophocle, mise en scène de Satoshi Miyagi, Cour d'honneur du Palais des Papes, Festival d'Avignon - du 6 au 12 juillet 2017.

1 En 2011, dans une interview, Xavier Douroux ${ }^{1}$ confiait, évoquant le nouveau bâtiment du Centre d'art Le Consortium ${ }^{2}$ qu'il a co-fondé et co-dirigé jusqu'à son décès, le 28 juin dernier: "Je crois beaucoup aux spectres des œuvres dans les expositions. Au fait qu'une œuvre, pour peu qu'elle ait eu un vrai rapport avec une salle, continue à la hanter. Tout cela est très lié à la mémoire (...) Que ce ne soit pas le lieu qui évolue, mais ce qui s'y passe, permet d'emporter plus aisément le souvenir et la conscience des œuvres. C'est une façon de se défier du spectaculaire, du contenant, pour privilégier la concentration sur le contenu. »Contenant vs contenu, spectaculaire vs concentration, permanence du lieu vs impermanence de l'œuvre: ces relations de tension opèrent dans tous les espaces, quel que soit le champ artistique. Pour autant, la question de la trace et du souvenir laissés est très peu évoquée dans le théâtre (on avancera l'hypothèse qu'elle passionnerait certains artistes comme le metteur en scène Claude Régy). L'interrogation prépondérante est plutôt celle de la pertinence d'un projet dans un lieu donné. Une réflexion épineuse, ignorée par le système majoritaire de fonctionnement du théâtre public: fondé sur la production/coproduction, celui-ci sous-entend qu'un spectacle, une fois créé, peut indifféremment jouer n'importe où. Ce qui n'est pas si évident ... 
2 Au premier rang des lieux où ces questions se mettent en jeu figure la Cour d'honneur du Palais des papes, à Avignon. Par ses volumes monumentaux, l'histoire à laquelle elle renvoie, mais aussi par la relation qu'elle noue avec le théâtre depuis 1947, la cour est un mythe. Métonymie du festival, lieu emblématique de la manifestation fondée par Jean Vilar, plus grande jauge de spectateurs d'Avignon, il s'y déploie le symbole du pouvoir du théâtre, comme de ses liens avec la puissance politique. Mais la cour est ingrate, et nombre d'artistes s'y sont cassé les dents, leur création ne passant pas la rampe. À titre d'exemple, La Mouette d'Anton Tchekhov montée par Arthur Nauzyciel en 2012 était comme écrasée par le bâtiment. Ce dernier impose au metteur en scène de prendre en compte ses volumes et de jouer subtilement avec eux.

Invité pour la deuxième fois au festival d'Avignon (sa première venue, en 2014, lui avait permis de présenter un Mahabharata dans la carrière de Boulbon), Satoshi Miyagi y présente Antigone. Comme il l'explique dans le programme de salle, le metteur en scène japonais précise avoir explicitement pris en compte «l'espace extrêmement particulier qu'est la Cour d'honneur. » C'est sur une scène envahie par l'eau et occupée par quelques rochers que les interprètes évoluent. Certains arpentent déjà le plateau lorsque les spectateurs prennent place, se déplaçant lentement avec une bougie tels des spectres vêtus de blanc. Cette procession lancinante dans cet espace magnétique installe le récit à venir comme un rituel, et rien, hormis un résumé de la pièce interprété en préambule à l'avant-scène sur un mode au farcesque appuyé, ne viendra contredire ce sentiment. Par la suite, tous, identiquement vêtus - de blanc, couleur du deuil au Japon - se meuvent avec solennité et alternent moments de jeu soutenus par la musique et épisodes choraux de danse ou de chants. Dans cette équipe, les personnages de la pièce de Sophocle ne se distinguent que par une perruque blanche. Enfin ... les corps des personnages. Car, et c'est là l'une des particularités de son travail, Satoshi Miyagi dissocie le corps du personnage de sa voix. Il fait jouer par des comédiens muets les intentions et actions, tandis que d'autres dans une semi-pénombre parlent pour eux. Il y a ainsi, d'un côté, évoluant sur les pierres sombres de la scénographie, largement éclairés, les personnages (Antigone condamnée à mourir pour avoir apporter les derniers sacrements à son frère Polynice, son fiancé Hémon qui mourra lui aussi, sa sœur Ismène, le roi Créon qui punit Antigone et reste sourd aux mauvais présages annoncés par Tirésias, etc.), dont le jeu volontairement stylisé et appuyé renvoie à toute une tradition du théâtre japonais. Il y a, de l'autre, la voix, dite par un comédien accroupi au sol, voix redoublée ou accentuée par des membres du chœur hormis Antigone qui par son choix d'enfreindre la loi royale demeure isolée. Et il y a la création lumières, qui surajoute à chaque rôle les ombres immenses des personnages. Projetées sur la façade du Palais des papes, celles-ci amplifient, décuplent les intentions des gestes et permettent à tous les spectateurs, où qu'ils soient placés, de les saisir. Mais paradoxalement, si cette Antigone est une réussite en termes d'appréhension de l'espace du Palais des papes, une découverte par sa façon de travailler avec toute une tradition du théâtre japonais (dont le Nô) en la réactualisant, la proposition demeure en-deçà de la pièce de Sophocle. Par cette dissociation des voix, des corps, cette surimpression des ombres, la mise en scène dilue son adresse, escamotant l'aspect tragique, violent de la pièce. Plus qu'une tragédie marquée par la fureur, c'est une cérémonie, ancrée dans une culture, une tradition japonaise, comme le souligne la venue par deux fois d'un homme en costume traditionnel - distribuant les perruques blanches aux comédiens jouant les personnages au début, déposant dans un rite mortuaire des bougies sur l'eau à la fin. Avec ce travail, Satoshi Miyagi inscrit cette 
histoire non pas dans un contexte funeste mais dans un mouvement cyclique, amené, peut-être, à se reproduire, ainsi que l'énonce la lente procession finale en cercle de tous les interprètes.

\section{NOTES}

1. Co-fondateur et co-directeur du Centre d'art Le Consortium, à Dijon, fondateur des Presses du Réel, Xavier Douroux était également - et entres autres choses - un enseignant génial par son érudition, la radicalité et l'exigence de sa pensée. Voir l'interview ici : https://issuu.com/media.pop/docs/novo_16/56

2. http://www.leconsortium.fr/ 